\title{
Optimal breast cancer screening strategies for older women: current perspectives
}

\author{
This article was published in the following Dove Press journal: \\ Clinical Interventions in Aging \\ 3 February 2016 \\ Number of times this article has been viewed
}

\author{
Dejana Braithwaite' \\ Joshua Demb' \\ Louise M Henderson ${ }^{2}$ \\ 'Department of Epidemiology and \\ Biostatistics, University of California, \\ San Francisco, CA, ${ }^{2}$ Department \\ of Radiology, University of North \\ Carolina, Chapel Hill, NC, USA
}

\begin{abstract}
Breast cancer is a major cause of cancer-related deaths among older women, aged 65 years or older. Screening mammography has been shown to be effective in reducing breast cancer mortality in women aged 50-74 years but not among those aged 75 years or older. Given the large heterogeneity in comorbidity status and life expectancy among older women, controversy remains over screening mammography in this population. Diminished life expectancy with aging may decrease the potential screening benefit and increase the risk of harms. In this review, we summarize the evidence on screening mammography utilization, performance, and outcomes and highlight evidence gaps. Optimizing the screening strategy will involve separating older women who will benefit from screening from those who will not benefit by using information on comorbidity status and life expectancy. This review has identified areas related to screening mammography in older women that warrant additional research, including the need to evaluate emerging screening technologies, such as tomosynthesis among older women and precision cancer screening. In the absence of randomized controlled trials, the benefits and harms of continued screening mammography in older women need to be estimated using both population-based cohort data and simulation models.
\end{abstract}

Keywords: aging, breast cancer, precision cancer screening

\section{Introduction}

Globally, breast cancer is the most commonly occurring cancer among women, comprising $23 \%$ of the $\sim 1.7$ million female cancers that are newly diagnosed each year. ${ }^{1,2}$ Approximately 6.2 million women were diagnosed with breast cancer in the last 5 years, making breast cancer the single most prevalent cancer around the globe. ${ }^{1}$ In the USA, breast cancer is responsible for most new cases of cancer among women with an estimated $29 \%$ of new cancer cases and $14 \%$ of cancer deaths in $2014 .^{2}$ Approximately $41 \%$ of all incident breast cancers and $57 \%$ of all breast cancer deaths occur among women aged 65 years and older. ${ }^{3}$

The incidence of breast cancer in the USA generally increases until 80 years of age, at which point the incidence begins to decrease, possibly due to lower rates of screening, the mammographic detection of cancers before 80 years of age, or incomplete detection. ${ }^{4}$ Screening mammography, the only population-based method for the early detection of breast cancer, has been shown to be effective in reducing breast cancer mortality in women aged 50-74 years. ${ }^{5,6}$ Yet, there is no evidence regarding the effectiveness of screening mammography in women aged 74 years and older. Diminished life expectancy that occurs with aging decreases the probability of a screening benefit and likely increases the risk of harms. ${ }^{7}$ Because of large heterogeneity in comorbidity status and life expectancy among older women, aged 65 years or older, a continuing controversy exists over screening mammography in this population..$^{8,9}$
Correspondence: Dejana Braithwaite Department of Epidemiology and Biostatistics, Mission Hall: Global Health \& Clinical Sciences Building, University of California, 550 16th Street, 2nd Floor, PO Box No 0560, San Francisco, CA 94I43, USA Tel +l 4I5 5148019

Email dejana.braithwaite@ucsf.edu 
The consequences of screening older women have not been well described, especially in relation to life expectancy. Randomized trials of screening mammography cannot provide the evidence because the trials excluded women older than 75 years and those with significant comorbidity. ${ }^{10}$

The impact of new imaging technologies on screening mammography outcomes in older women is not well understood. Although routine screening with two-dimensional (2D) digital mammography is the primary means of early breast cancer detection, the use of newer imaging technologies, such as digital breast tomosynthesis (DBT, also referred to as 3D mammography) is diffusing rapidly into clinical practice. ${ }^{11}$ In recent studies, the addition of DBT to $2 \mathrm{D}$ digital mammography resulted in a decrease in recall rates and an increase in cancer detection rates, when compared with 2D digital mammography alone. ${ }^{12-17}$ Given that these findings point to significant improvements in breast cancer screening outcomes with DBT, it will be important to include women in older age ranges in future studies of DBT.

In this review, we summarize the evidence and current perspectives regarding the utilization of screening mammography and performance and outcomes in older women and highlight evidence gaps in this field.

\section{Screening mammography utilization in older women}

Several guidelines support screening mammography in older women unless a woman's comorbid conditions limit life expectancy (Table 1). In women aged 70 years and older, the World Health Organization recommendation only specifies that well-resourced settings with the infrastructure to create population-based programs should provide screening. ${ }^{18}$ The US Preventive Services Task Force (USPSTF) updated their guidelines in 2009 to recommend biennial, rather than yearly screening mammography until 74 years of age but concluded that evidence was insufficient to make recommendations for women aged 75 years and older. ${ }^{10,18}$ Recently revised breast cancer screening recommendations from the American Cancer Society (ACS) are for regular screening mammography for women at an average risk of developing breast cancer beginning at 45 years of age and continuing after 70 years of age amongst women who are in good health. ${ }^{19}$ The National Cancer Institute is reevaluating its past recommendations in light of the USPSTF recommendations and supporting further research. ${ }^{10}$ Both the ACS and the USPSTF guidelines state that screening in older women should be considered on an individual basis through the evaluation of potential benefits and risks posed by the mammogram in relation to their current health conditions and predicted life expectancy.

In the USA, screening mammography attendance rates among older women are generally high. For example, $\sim 73 \%$ of US women aged 75 years or older reported having undergone screening mammography in the 2010 US Behavioral Risk Factor Surveillance System in the last 2 years. ${ }^{20}$ According to data from the 2013 National Health Interview Survey, $75.3 \%$ of women aged $65-74$ years and $56.5 \%$ of women aged 75 years and older self-reported screening mammography use in the last 2 years. ${ }^{21}$ Crucially, screening mammography is also commonly reported among older US women in poor health in the National Health Interview Survey ${ }^{22-24}$ and in the US Breast Cancer Surveillance Consortium. ${ }^{25}$ Thus, many older women undergo screening mammography without evidence of benefits from screening.

\section{Screening mammography utilization by comorbidity and functional status}

In older women, comorbid conditions and diminished life expectancy may influence a physician's decision to recommend mammography or a woman's decision to undergo screening. ${ }^{26,27}$ Table 2 provides a summary of studies that evaluated the association between comorbidity and screening mammography utilization. Several of the studies evaluating comorbidity and screening utilization reported that a higher

Table I Guideline recommendations about screening mammography in older women

\begin{tabular}{|c|c|c|c|}
\hline USPSTF guidelines & ACS guidelines & ACR guidelines & AGS guidelines \\
\hline $\begin{array}{l}\text { Offer biennial screening to } \\
\text { women aged } 50-74 \text { years. } \\
\text { Evidence is insufficient to } \\
\text { recommend for or against } \\
\text { screening in women }>74 \text { years } \\
\text { of age. "I" statement*. The } \\
\text { Task Force encourages more } \\
\text { research on the topic. }\end{array}$ & $\begin{array}{l}\text { Offer screening to women } \\
\text { aged } \geq 45 \text { years and } \\
\text { continue as long as a woman } \\
\text { is in good health and has life } \\
\text { expectancy of } \geq 10 \text { years. }\end{array}$ & $\begin{array}{l}\text { Offer annual screening to } \\
\text { women aged } \geq 40 \text { years } \\
\text { and continue as long as a } \\
\text { woman is in good health. }\end{array}$ & $\begin{array}{l}\text { Offer screening to women aged } \leq 85 \text { years } \\
\text { who have life expectancy of } \geq 5 \text { years and } \\
\text { for healthy women aged } \geq 85 \text { years who } \\
\text { have excellent functional status or who feel } \\
\text { strongly about the benefits of screening } \\
\text { (no screening frequency specified). }\end{array}$ \\
\hline
\end{tabular}

Notes: *Current evidence is insufficient to address benefits and harms of breast cancer screening in women $>74$ years of age.

Abbreviations: ACR, American College of Radiology; ACS, American Cancer Society; AGS, American Geriatrics Society; USPSTF, US Preventive Services Task Force. 







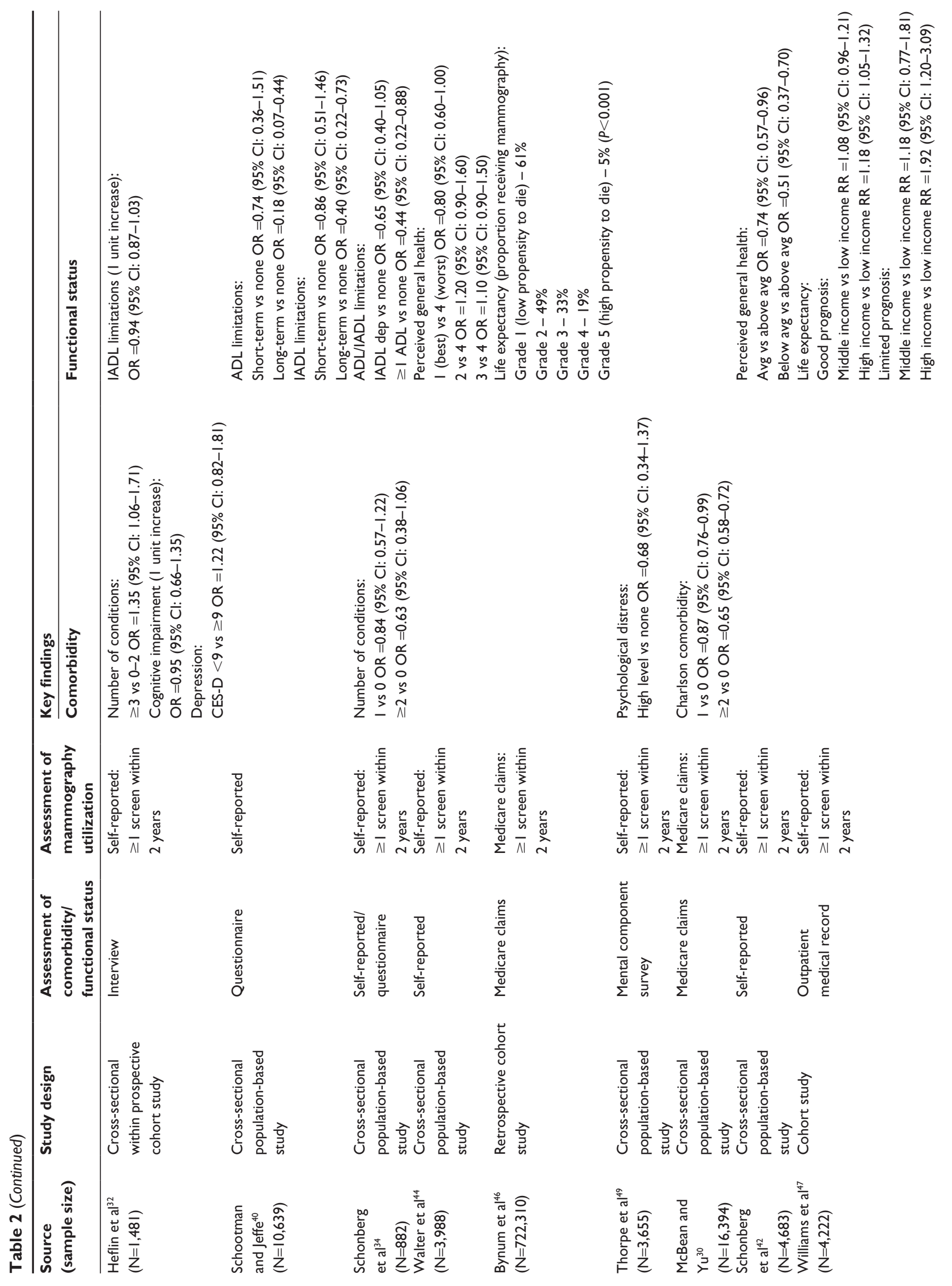



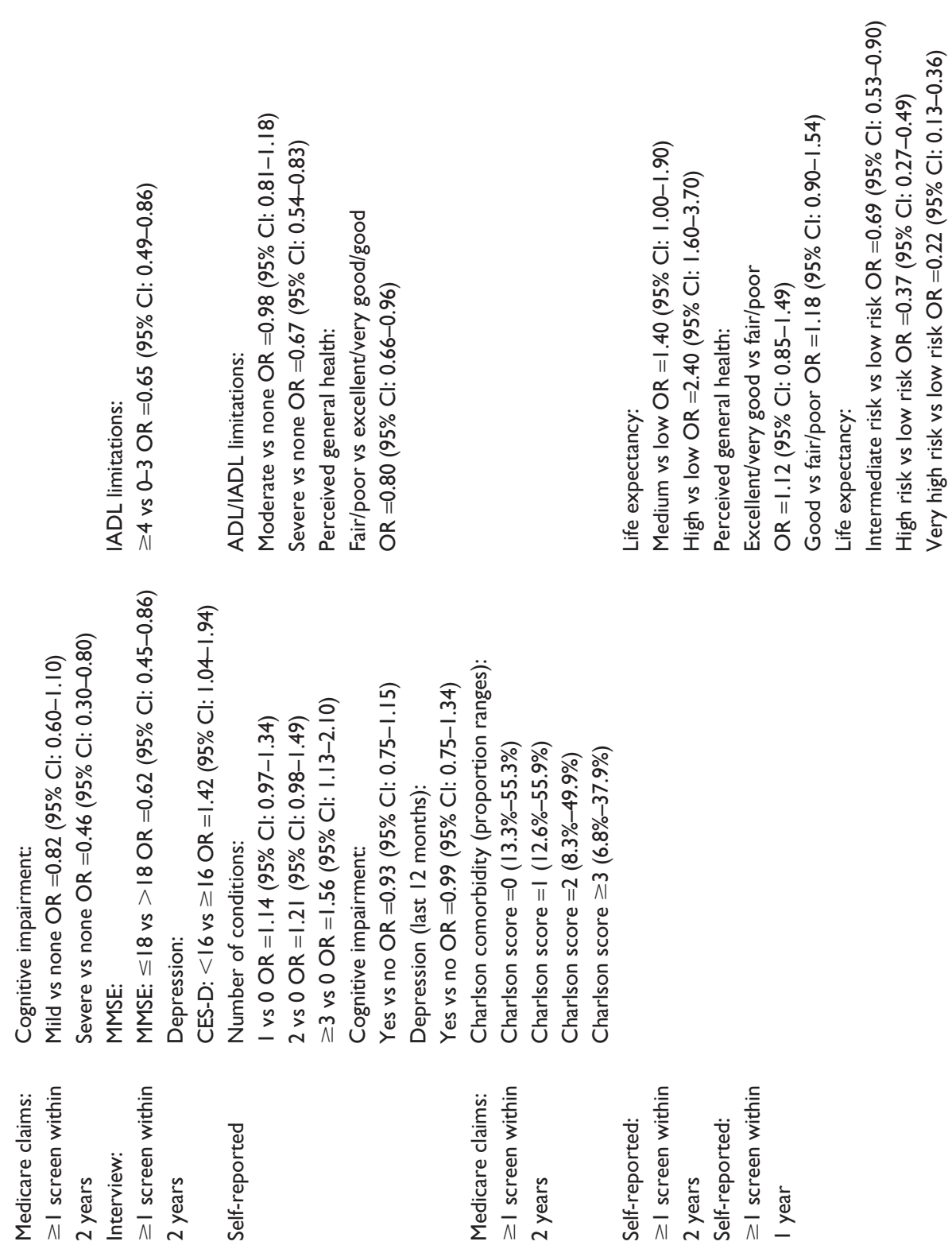

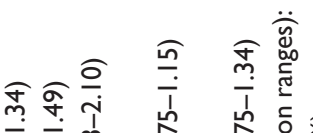

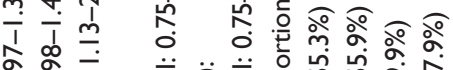

O.

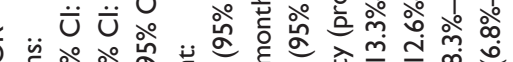

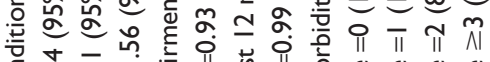

至

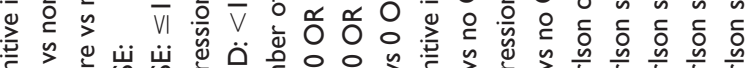

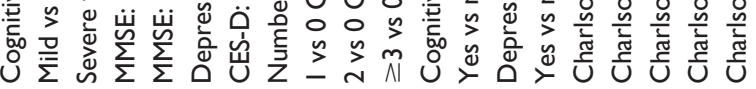
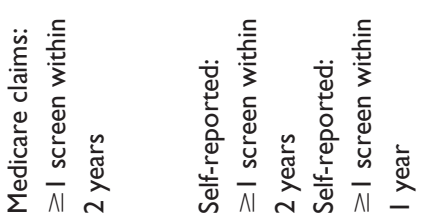

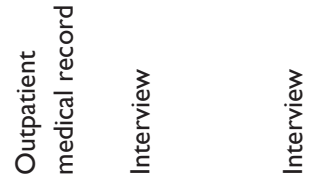
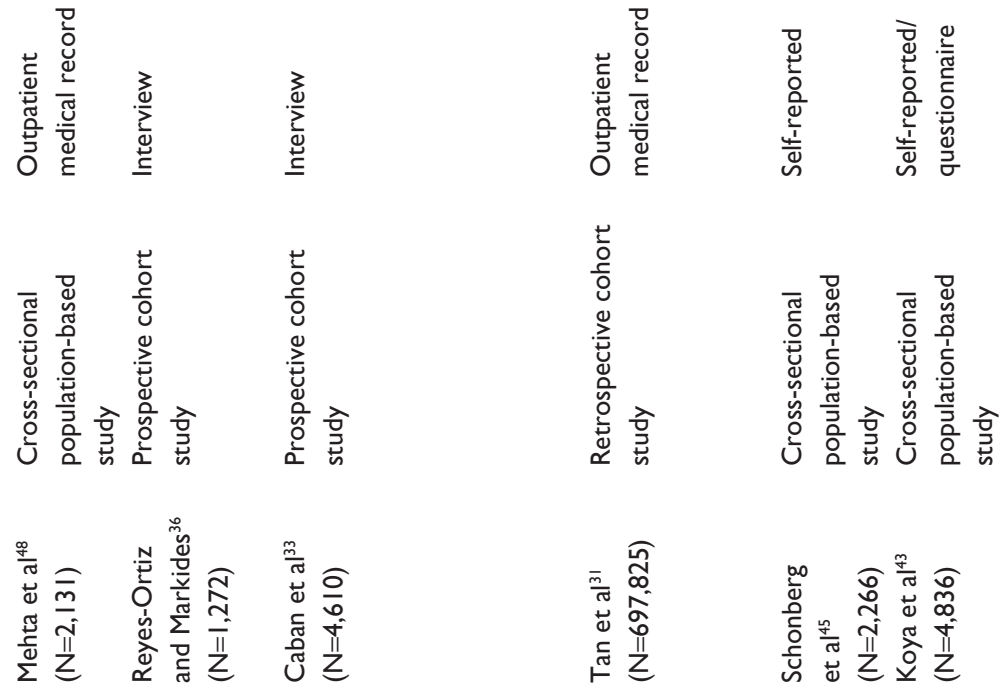

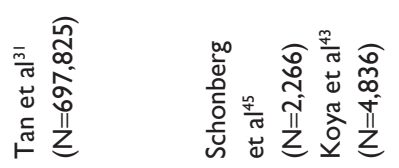


Charlson comorbidity score was associated with lower screening utilization. ${ }^{28-31}$ For example, women with Charlson scores of $\geq 2$ were found to have a $35 \%$ reduction in the odds of mammography utilization (odds ratio [OR]: 0.65, 95\% confidence interval $[\mathrm{CI}]: 0.58-0.72) .{ }^{30}$ Conflicting evidence exists regarding the impact of the total number of comorbid conditions on screening use, with two studies finding that higher numbers of comorbid conditions increased screening mammography utilization, ${ }^{32,33}$ whereas two other studies reported an inverse association. ${ }^{34,35}$ This variance may reflect the use of different sums of comorbid conditions.

Studies evaluating the associations between cognitive impairment, depression, and screening mammography utilization have generally shown inconclusive results (Table 2) In a study of Mexican American women aged 75 years and older that measured cognitive impairment (using the minimental state examination [MMSE]), lower MMSE scores were associated with decreased odds of screening utilization (OR: 0.62, 95\% CI: 0.45-0.86). ${ }^{36}$ Moreover, the same study reported that increased depressive symptoms, as reflected by the Center for Epidemiological Studies-Depression (CES-D) scale, were associated with increased screening mammography utilization. ${ }^{36}$ However, other studies measuring cognitive impairment with MMSE and depression with CES-D scale in more diverse populations found equivocal results. ${ }^{32,33,35,37,38}$

Studies of functional limitations have generally found an inverse association with screening utilization (Table 2). Specifically, activities of daily living (ADL) limitations were associated with decreased screening mammography utilization, ${ }^{22,37-40}$ with one study in 2003 finding more significant decreases in utilization in women older than 70 years (OR: 0.18, 95\% CI: 0.07-0.44). ${ }^{40}$ Similar results were found with instrumental activities of daily living (IADL) limitations, ${ }^{32,36,38,40}$ since long-term IADL limitations identified by reporting limitations at both visits - were more strongly associated with decreased mammography utilization (OR: 0.40, 95\% CI: 0.22-0.73). ${ }^{40}$ When considering scales using both ADL and IADL measurements, having severe limitations led to significant decreases in odds of screening mammography. ${ }^{29,33,34}$

In general, women's perceptions of their general health were not statistically significant predictors of change in screening mammography utilization (Table 2). Of the seven studies measuring perceived general health in older women, ${ }^{29,33,37,41-44}$ only two found a significant positive association between declining perceived health status and screening mammography utilization. ${ }^{33,42}$ Life expectancy measured by a prognostic index was a strong predictor of screening mammography utilization in older women, with four studies indicating that women with a higher risk of mortality had lower odds of screening mammography. . $^{39,43,45,46}$ Notably, Koya et al found a nearly $80 \%$ decrease in odds of mammography utilization in women in the lowest life expectancy group (OR: 0.22, 95\% CI: 0.13-0.36). ${ }^{43}$ Moreover, in a study that used a life expectancy index with income as a stratifying covariate, women with higher incomes and longer life expectancy (relative risk [RR]: 1.18, 95\% CI: 1.05-1.32) or higher incomes and limited life expectancy (RR: 1.92, 95\% CI: 1.20-3.09) had increased utilization of screening mammography than their counterparts with lower incomes. ${ }^{47}$

There is paucity of data examining the association between comorbidity or life expectancy and screening mammography utilization in older women outside of the USA. Of note, many of the aforementioned studies employed as the main outcome claims (or health insurance-derived) data d0,31,37-39,46,48 $^{30}$ or self-reported mammography utilization, ${ }^{32-34,40,42,44,45,47,49}$ with the latter being more likely to result in potentially biased effect estimates.

In summary, there is compelling evidence that older women with a greater comorbidity burden and poorer functional status are less likely to undergo screening mammography, particularly among studies that employed standardized comorbidity measures. ${ }^{28-31}$ Moreover, diminished life expectancy was also found to be inversely associated with mammography utilization. ${ }^{39,43,45,46}$ Although perceived general health was found to be an inconclusive predictor of screening utilization, ${ }^{29,33,37,41-44}$ further research on the impact of life expectancy indicators may enhance our understanding of screening mammography utilization in older women.

\section{Screening mammography performance in older women}

Overall, there is limited evidence regarding screening mammography performance in older women. Hitherto, two studies have explicitly examined screening mammography performance in older US women. ${ }^{50,51}$ A 2011 study by Sinclair et al evaluated the accuracy and cancer detection rate among 403,448 mammograms (the majority of which were captured with film-screen mammography) for women aged 50-101 years living in Vermont. ${ }^{50}$ Interestingly, screening mammography performance improved with age in this study; when compared to women aged 50-59 years, those aged $70-79$ years had an increase in sensitivity $(77.3 \%-80.4 \%)$, 
specificity $(98.7 \%-99.0 \%)$, positive predictive value $(22.2 \%-37.6 \%)$, and cancer detection rate $(3.7 / 1,000$ $6.2 / 1,000$ mammograms). ${ }^{50}$ The relationship between age and performance measures was not influenced by potential confounders of body mass index, breast density, education, race, ethnicity, family history of breast or ovarian cancer, personal history of ovarian cancer, current or prior use of hormone therapy, and age at menopause or menarche.

The second study in USA, published in 2015, utilized the national Breast Cancer Surveillance Consortium data from 296,496 full-field digital screening mammograms among women aged 65 years and older to assess performance. ${ }^{51}$ Of note, the performance measures in this study were also stratified by the Breast Imaging Reporting and Data Systems' breast density values to determine if breast density rather than age was affecting mammography performance. Similar to the 2011 study, the specificity, positive predictive value, and cancer detection rate of digital screening mammography improved significantly with increasing age. In contrast to the 2011 study, ${ }^{50}$ the sensitivity of digital screening mammography did not increase with age and was $88.3 \%$ overall. The recall rate, which was not examined in the earlier study, ${ }^{50}$ decreased significantly from $8.4 \%(95 \% \mathrm{CI}: 7.8 \%-8.0 \%)$ in women aged $65-69$ years to $7.3 \%$ (95\% CI: $6.9 \%-7.8 \%)$ in women aged 85 years and older. Adjusted models showed similar improvements with increased age, suggesting that both age and breast density impact the recall rate, specificity, positive predictive value, and cancer detection rate. Of note, this study evaluated digital mammography because of its widespread utilization in the USA and did not consider film mammography; the cost-effectiveness of digital mammography compared to film mammography in older women has not been established. ${ }^{52}$

Because screening mammography programs outside the USA do not typically include women older than 70 years or 74 years, there is limited evidence on the performance of screening mammography at the 5- or 10-year age-groups necessary to evaluate performance in older women. The Ontario Breast Screening Program that includes women aged 50-59 years, 60-69 years, and 70-74 years and reports performance measures for these groups reported significant increases in cancer detection rate (CDR) and positive predictive values with increasing age, and a significant decrease in the recall rate with increasing age. ${ }^{53}$

Results from both US studies ${ }^{50,51}$ show that as age increased, the proportion of invasive versus ductal carcinoma in situ (DCIS) cases increased, with the exception of women aged 90-101 years in the Vermont study; approximately
$75 \%-81 \%$ of cancers detected in older women were invasive. In both studies, ${ }^{50,51}$ the proportion of cases with positive nodes decreased with increasing age. Tumors detected in the era of film-screen mammography showed a positive association of age and estrogen receptor-positive status, with the proportion of estrogen receptor-positive increasing with increasing age.$^{51}$ However, in the digital screening era, as age increased, the proportion of lower grade tumors increased. ${ }^{52}$ Neither study found a significant association of tumor stage with age. ${ }^{50,51}$ Moreover, a study by SmithBindman et al in 2000 found that women aged 66-79 years who underwent screening mammography had a decreased risk of detecting metastatic breast cancer. ${ }^{54}$ Of note, neither of these aforementioned studies examined screening mammography performance in the context of comorbidity or life expectancy. ${ }^{50,51}$

\section{Screening mammography outcomes in older women}

Since rates of clinically indolent tumors and DCIS increase with age, older women are more likely to be harmed from overdiagnosis, ${ }^{55}$ defined as detection of tumors by screening that would not become clinically apparent during a woman's lifetime or would not affect overall survival. Given the steeper rise in competing causes of mortality in women older than 74 years, evidence suggests that rates of overdiagnosis are likely to be greater for older women than younger women. ${ }^{55,56}$ Screening tests can have immediate harmful consequences and the long-term benefits of screening may not be realized in women with a short life expectancy. ${ }^{26,27,57-59}$ The most important benefit of screening mammography in older populations is an improvement in life expectancy, while the harms include false-positive results and overdiagnosis. ${ }^{7}$ Given the increasing comorbidity burden and attendant decline in life expectancy, some older women are unlikely to have a favorable benefit/harm ratio..$^{58,60}$

The currently available evidence regarding the impact of comorbidity and health status on screening mammography outcomes consists of four observational ${ }^{25,61-63}$ and three decision models ${ }^{64-66}$ because no randomized trials included women older than 74 years. It is important to recognize that observational data are subject to selection bias as well as lead-time and length bias. In observational studies evaluating screening mammography, the study populations of older women have self-selected to undergo screening mammography and are likely to be healthier than the general US population. ${ }^{64-66}$ Both cohort studies and decision analytic models ${ }^{25,61-66}$ found that screening benefits decreased with 
increasing age and comorbidity burden. Thus, the balance of benefits versus harms varies according to comorbidity and age, which underscores the need for evidence to develop life expectancy-based screening strategies.

\section{Benefits of screening mammography in older women}

Only one cohort study has so far evaluated mortality as a benefit of breast cancer screening. ${ }^{63}$ In the study by McPherson et al, ${ }^{63}$ which included 5,186 women aged 65 years and older diagnosed with breast cancer between 1986 and 1994 through the Upper Midwest Tumor Registry system, women's comorbidity was assessed via the Charlson score. ${ }^{67}$ In this study, women aged 65 years and older with no or moderate comorbidity and mammographically detected tumors were found to be at reduced risk of breast cancer death compared to those with clinically detected tumors (Table 3). ${ }^{63}$ In addition, among women with severe comorbidity, as defined by a Charlson score of $\geq 3$, screening mammography was associated with reduced breast cancer mortality among women aged 70-74 years, but not in those younger than 70 years or older than 74 years. ${ }^{63}$

Although detection of early stage disease at diagnosis has been utilized as a marker of screening benefit, this may not necessarily represent a benefit in older women with indolent tumors. Of the three cohort studies that evaluated the risk of early versus advanced tumor stage, ${ }^{25,61,62}$ two - Braithwaite et $\mathrm{al}^{61}$ and Yasmeen et $\mathrm{al}^{25}$ - used data from the US Breast Cancer Surveillance Consortium linked to Medicare insurance claims data from 1999 to 2006, to evaluate comorbidities in the 2 years before screening mammography. In another cohort study, Fleming et al merged data from the Surveillance, Epidemiology and End Results program with Medicare insurance claims for 17,468 women diagnosed with breast cancer between 1993 and 1995. ${ }^{62}$ Heterogeneous measures of comorbidity were utilized in these three studies: Braithwaite et $\mathrm{al}^{61}$ employed the Charlson comorbidity score while Fleming et $\mathrm{al}^{62}$ and Yasmeen et $\mathrm{al}^{25}$ reported on 24 individual conditions, and severity-based categorizations of comorbidity, respectively. Yasmeen et al found that overall rates of advanced breast cancer (per 1,000 mammograms) were lower among women with no comorbidity than among those with stable comorbidity in annually and biennially screened women and for those that received their first screen (Table 3). ${ }^{25}$ However, among women who had prior mammography within 4-18 months of cancer diagnosis, the rates of advanced-stage cancer were higher among those with either stable or unstable comorbidities than among those without comorbidities. ${ }^{25}$ In contrast, Braithwaite et $\mathrm{l}^{61}$ reported that adverse tumor characteristics, including advanced stage, did not differ significantly by the Charlson score or screening interval. Moreover, Fleming et $\mathrm{al}^{62}$ reported that women with cardiovascular disease, musculoskeletal disorders, mild-tomoderate gastrointestinal disease, and nonmalignant benign breast disease had a $13 \%, 7 \%, 14 \%$, and $24 \%$ lower odds, respectively, of being diagnosed with advanced breast cancer, while those with diabetes, other endocrine disorders, psychiatric disorders, and hematologic disorders had increased odds of advanced stage diagnosis by $19 \%, 11 \%$, $20 \%$, and $19 \%$, respectively, compared to women without these comorbidities.

Consistent with observational data, decision analyses confirm that women aged 65 years or older are less likely to benefit from screening, particularly if they have severe comorbidity, ${ }^{68}$ and propose a comorbidity-dependent cessation age. ${ }^{65}$ Moreover, another decision analytic model reported minimal quality-adjusted life expectancy for women aged 85 years and older with average health or mild comorbidity and losses in quality-adjusted life expectancy for women with severe comorbidity. ${ }^{64}$ Specifically, two decision analyses, Mandelblatt et $\mathrm{al}^{68}$ and Lansdorp-Vogelaar et al, ${ }^{65}$ employed well-established, independently developed Cancer Intervention and Surveillance Modeling Network models, with each model simulating the life histories of large US cohorts, and assessing the underlying disease in the presence and absence of screening. Relative life expectancy benefits of screening in older women according to comorbidity are shown in Table 3. In particular, Lansdorp et al compared the number needed to screen per life-year gained at different stopping ages and estimated threshold stopping ages according to the level of comorbidity, at which the number needed to screen per life-year gained was the same as that of mammography until 74 years of age for women of average comorbidity. ${ }^{65}$ Authors evaluated biennial screening mammography from 50 years of age to a cessation age ranging from 66 years to 90 years by simulating US cohorts of women who were 66-90 years old and alive in 2010 , and had no comorbidity, mild comorbidity (a history of myocardial infarction, acute myocardial infarction, ulcer, or rheumatologic disease), moderate comorbidity (the presence of vascular disease, cardiovascular disease, paralysis or, diabetes), or severe comorbidity (the presence of AIDS, mild or severe liver disease, chronic obstructive pulmonary disease, chronic renal failure, dementia, or congestive heart failure), as well as comparison cohorts of average comorbidity aged 


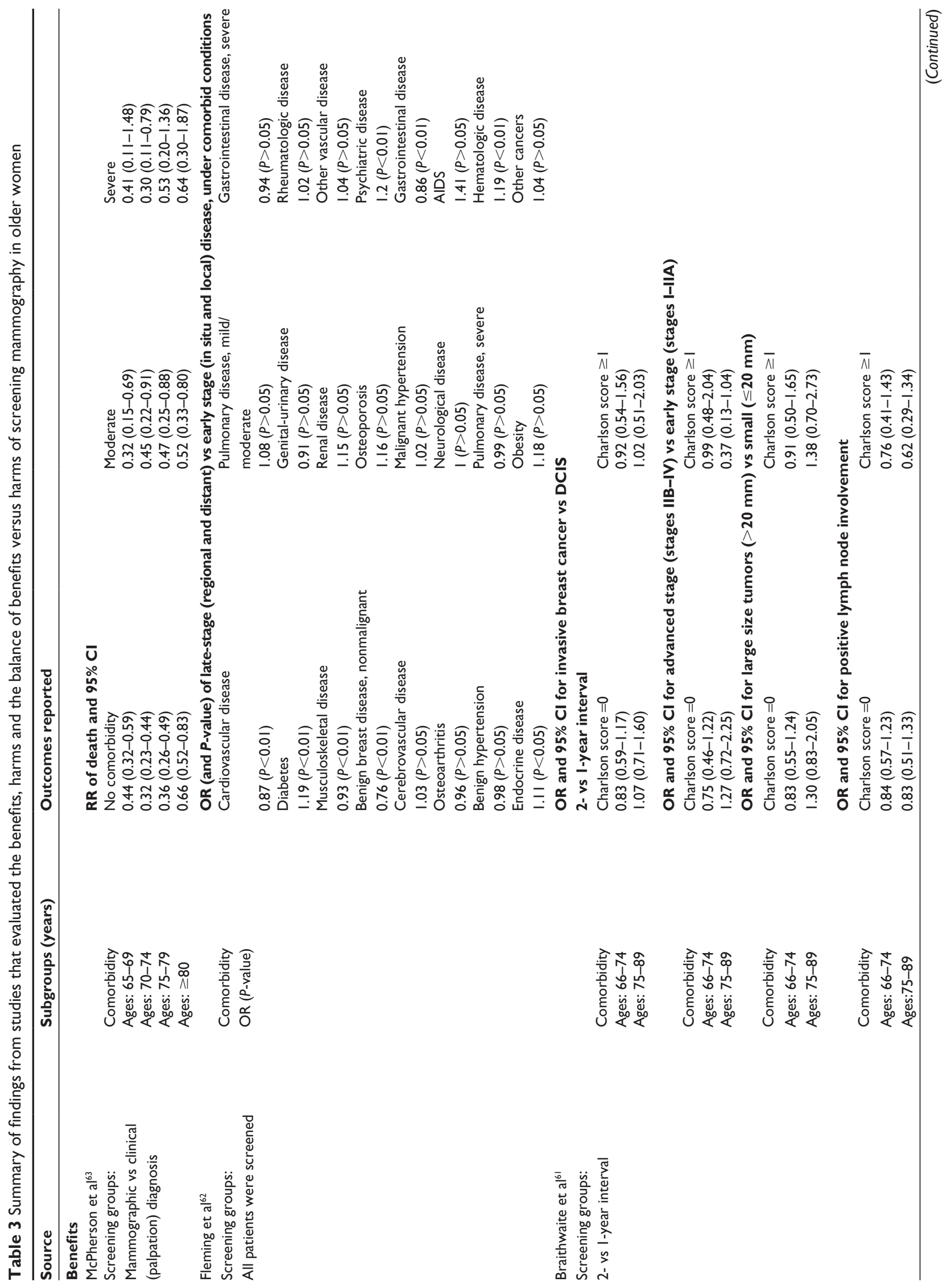




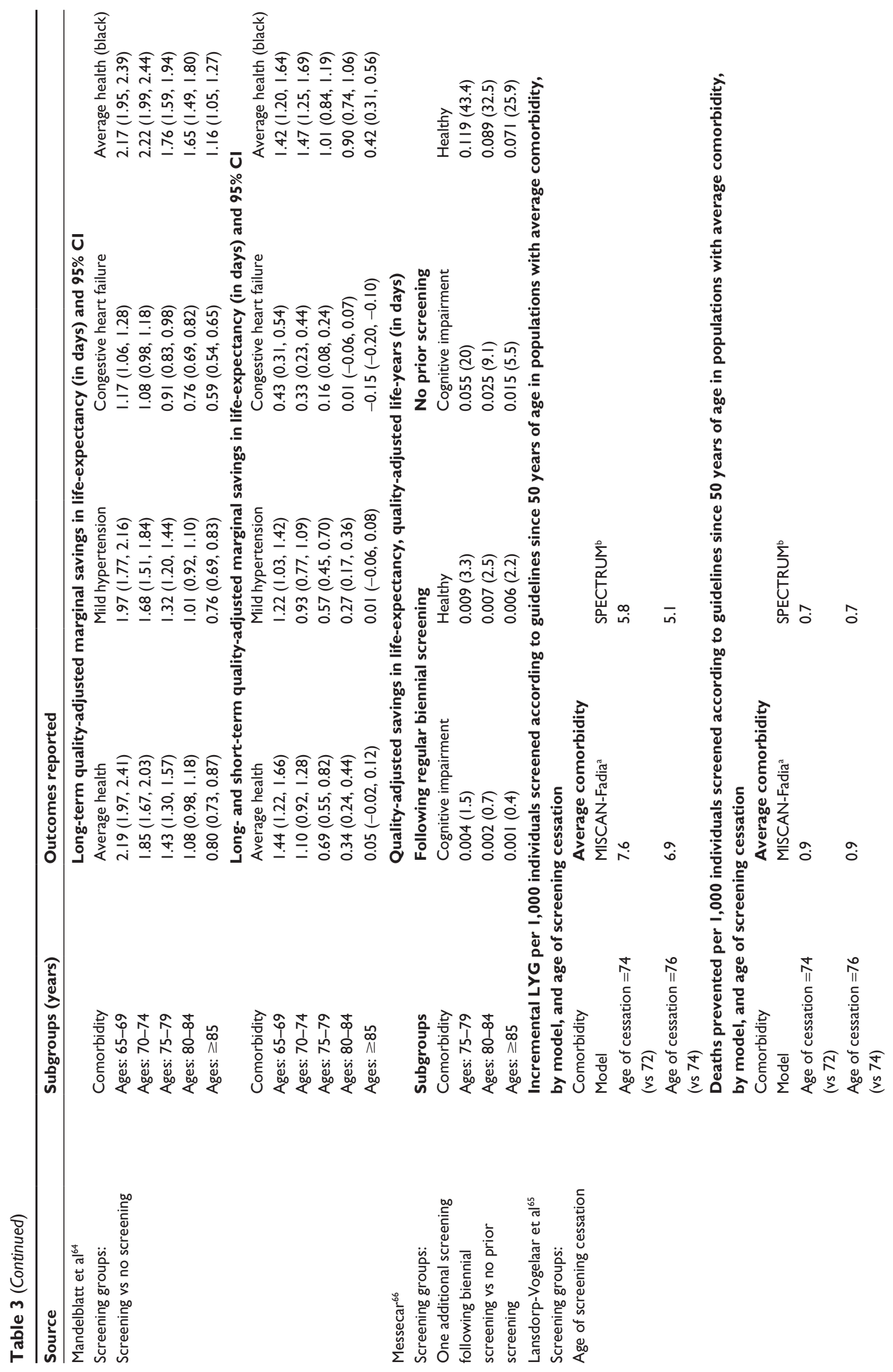




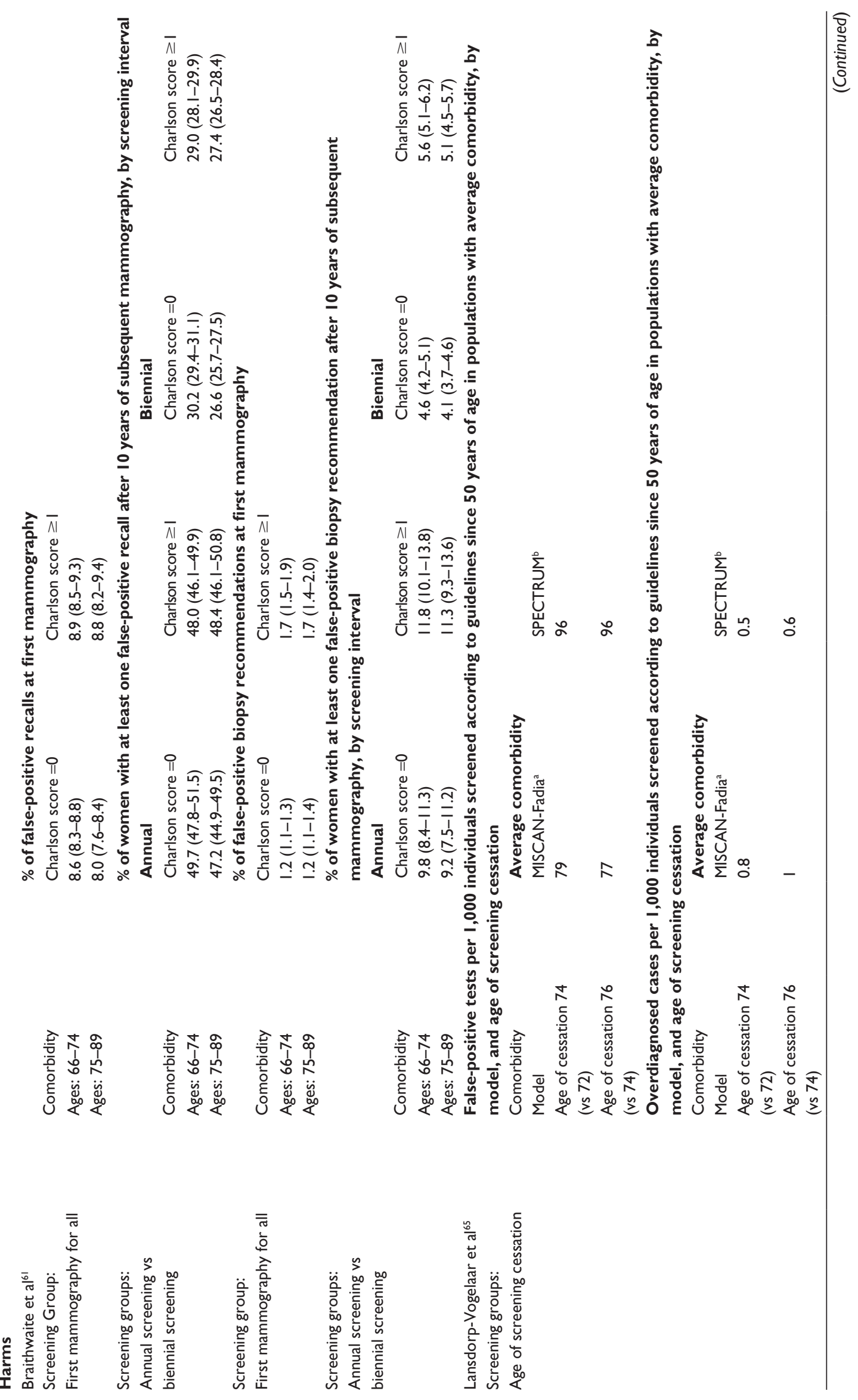


74 years and 76 years. In this study, Lansdorp et al found that breast cancer screening through 74 years of age resulted in a number needed to screen to gain 1 life-year among women with no comorbidity of 117-149 across models, which was lower than that in the entire population with average comorbidity; cessation of screening at 76-78 years of age among women with no comorbidities was estimated to yield the same number needed to screen to gain 1 life-year as cessation at 74 years of age in the entire population. ${ }^{65}$ Finally, this study points to the benefits of biennial mammography across models until median ages of 76-78 years, 74 years, 70-72 years, and 64-68 years for women with no comorbidity, mild comorbidity, moderate comorbidity, and severe comorbidity, respectively. ${ }^{65}$ In hypothetical cohorts examining benefits of biennial screening in terms of life-years, Mandelblatt et a ${ }^{64}$ found that long- and short-term quality-adjusted savings in life expectancy from screening compared to a nonscreening strategy were greater for older women with mild hypertension than for those with heart disease, and the benefit in both groups decreased with increasing age (Table 3). Finally, in another decision analysis examining three hypothetical cohorts of women aged $75-79$ years, 80-84 years, and $\geq 85$ years with and without cognitive impairment, Messecar tested the gain in quality-adjusted life-years in two models for each group assuming no prior screening versus continued biennial screening. In this study, ${ }^{66}$ all older women benefited from biennial screening mammography, although among women with no prior screening, the gain in quality-adjusted life-years was lower among cognitively impaired women (20 days, 9.1 days, and 5.5 days for age-groups 75-79 years, 80-84 years, and $\geq 85$ years, respectively) than their healthy counterparts (43.4 days, 32.5 days, and 25.9 days for age-groups 75-79 years, $80-84$ years, and $\geq 85$ years, respectively). ${ }^{66}$

The aforementioned benefits should be considered in conjunction with reported harms of screening in older women.

\section{Harms of screening mammography in older women}

There are evidence gaps regarding the harms of screening mammography in older women according to comorbidity and life expectancy; ${ }^{61,65}$ a summary of studies that have hitherto addressed this question is shown in Table 3. In the US Breast Cancer Surveillance Consortium cohort study that evaluated the harms of screening mammography, Braithwaite et al reported that the 10-year cumulative probability of a false-positive mammography result was higher among annual screeners than biennial screeners irrespective of comorbidity: $48.0 \%$ (95\% CI: $46.1 \%-49.9 \%$ ) of annual screeners aged 66-74 years 
had a false-positive result compared with $29.0 \%$ (95\% CI: $28.1 \%-29.9 \%$ ) of biennial screeners. ${ }^{61}$ In a decision-analytic study evaluating the harms of screening, Lansdorp-Vogelaar et $\mathrm{al}^{65}$ showed that ending screening at 74 years versus 72 years of age resulted in 96 more false-positive tests and 0.5 more overdiagnoses per 1,000 screening tests (Table 3). In examining the balance of benefits versus harms from screening mammography, Lansdorp-Vogelaar et $\mathrm{al}^{65}$ also assessed numbers needed to screen in relation to life-years gained and estimated that extending breast cancer screening from the age of 72 years until 74 years of age among individuals with average comorbidity, required screening 132-174 women to gain 1 life-year; continuing screening until 76 years of age required an additional 146-198 women to be screened to gain 1 life-year. ${ }^{65}$

Another simulation model indicated that personalized screening based on individual risk that is measured as a function of age, breast density, history of breast biopsy, family history of breast cancer, and screening interval could potentially improve the balance of benefits versus harms among not only older but also younger women, where low-risk women could stop screening or continue to be screened at longer intervals, thereby reducing false-positive results. ${ }^{69}$

\section{Decision-making regarding screening mammography among older women}

Communication about potential benefits and harms to older women in their 70 s and 80 s also poses a challenge, given the limited available evidence..$^{7,60,70-72}$ In light of this uncertainty, clinical decisions about undergoing mammography in older populations would likely benefit from adopting life expectancy-based screening. A recent metaanalysis of survival data from population-based, randomized controlled trials comparing populations screened and not screened for breast cancer reported that it took 10.7 years (4.4-21.6 years) on average across included studies, before one death from breast cancer was prevented for 1,000 women screened; hence, this study concluded that screening for breast cancer should be targeted to women with a life expectancy $>10$ years. ${ }^{57}$ To this end, it will be important for primary care physicians to adopt prognostic tools that provide estimates of women's risk of 10 -year mortality, ${ }^{73}$ since such tools may facilitate informed decisions about screening.

A prognostic tool developed by Cruz et $\mathrm{l}^{73}$ based on data from the Health and Retirement Survey, a nationally representative cohort of community-dwelling US adults $>50$ years, is a 12-item mortality index that calculates an estimate of 10-year mortality based on age, sex, tobacco use, body mass index, diabetes, nonskin cancer, chronic lung disease, heart failure, and ADL (difficulty bathing, difficulty managing finances, difficulty walking several blocks, and difficulty pushing/pulling objects, etc). Application of valid prognostic tools in primary care settings may identify women with a low versus high risk of 10-year mortality that would and would not benefit from screening mammography, respectively. Recently developed decision aids show promise for counseling older women about the benefits and harms of screening mammography ${ }^{74}$ and may help overcome the challenges of implementing life expectancybased screening strategies in clinical practice.

\section{Conclusion and future directions}

In summary, screening mammography may be beneficial to older women if they have life expectancy of at least 10 years. Optimizing the screening strategy will involve a careful balance of benefits versus harms and life expectancy-based screening strategies. While the balance of benefits versus harms may be favorable for women up to 69 years of age and perhaps even up to 74 years of age with biennial screening, there is little evidence to support annual screening in older populations. Consistent with this, the updated USPSTF guidelines recommend biennial screening for women aged 66-74 years, but there are no explicit recommendations for women aged 75 years and older because of insufficient evidence. To better target populations who will benefit from screening, the National Cancer Institute has launched a new precision-based cancer screening initiative. ${ }^{75}$ With the aging of the population, it will be increasingly important to evaluate life expectancy-based screening by identifying women with sufficient life expectancies to benefit from screening, while minimizing harms associated with false-positive results and overdiagnosis among women who will not live long enough to benefit.

This review has identified many areas related to screening mammography in older women that need additional research. For example, there is a paucity of research evaluating emerging screening technologies such as tomosynthesis among older women. Without randomized controlled trials, the benefits and harms of continued screening mammography in older women will need to be estimated using a combination of cohort data and simulation models.

As pointed out in the recent $J N C I$ editorial, ${ }^{76}$ direct application of simulation models to the breast cancer screening policy and clinical practice remains a challenge. To address this gap and eschew the pseudoprecision that modeling can portray, ${ }^{76}$ it will be important to combine empirical evidence with modeling. Moreover, moving the field forward will necessitate modeling screening performance and mortality as a function of comorbidity, cognitive/physical functioning, 
and life expectancy as well as cost-effectiveness of different screening strategies according to these factors.

\section{Acknowledgment}

Dejana Braithwaite was supported by Grant \# 121891MRSG-12-007-01-CPHPS from the American Cancer Society for this research.

\section{Disclosure}

The authors report no conflicts of interest in this work.

\section{References}

1. Torre LA, Bray F, Siegel RL, Ferlay J, Lortet-Tieulent J, Jemal A. Global cancer statistics, 2012. CA Cancer J Clin. 2015;65:87-108.

2. Siegel R, Naishadham D, Jemal A. Cancer statistics, 2012. CA Cancer J Clin. 2012;62:10-29.

3. Howlader N, Noone A, Krapcho M, et al. SEER Cancer Statistics Review, 1975-2009 (Vintage 2009 Populations). Bethesda, MD: 2012.

4. Alteri R, Barnes C, Burke A, et al. Breast Cancer Facts \& Figures 2013-2014. Atlanta: American Cancer Society; 2013.

5. Marmot MG, Altman DG, Cameron DA, Dewar JA, Thompson SG, Wilcox M. The benefits and harms of breast cancer screening: an independent review. Br J Cancer. 2013;108:2205-2240.

6. Gotzsche PC, Nielsen M. Screening for breast cancer with mammography. Cochrane Database Syst Rev. 2006;(4):CD001877.

7. Braithwaite D, Mandelblatt JS, Kerlikowske K. To screen or not to screen older women for breast cancer: a conundrum. Future Oncol. 2013;9(6):763-766.

8. Barratt A, Howard K, Irwig L, Salkeld G, Houssami N. Model of outcomes of screening mammography: information to support informed choices. BMJ. 2005;330(7497):936.

9. Cohen HJ. The cancer aging interface: a research agenda. J Clin Oncol. 2007;25(14):1945-1948.

10. US Preventive Services Task Force. Screening for breast cancer: U.S. Preventive Services Task Force recommendation statement. Ann Intern Med. 2009;151:716-726,W-236.

11. Hardesty LA. Issues to consider before implementing digital breast tomosynthesis into a breast imaging practice. AJR Am J Roentgenol. 2015;204:681-684.

12. Rose SL, Tidwell AL, Bujnoch LJ, Kushwaha AC, Nordmann AS, Sexton R. Implementation of breast tomosynthesis in a routine screening practice: an observational study. AJR Am J Roentgenol. 2013;200: 1401-1408.

13. Skaane P, Bandos AI, Gullien R, et al. Prospective trial comparing fullfield digital mammography (FFDM) versus combined FFDM and tomosynthesis in a population-based screening programme using independent double reading with arbitration. Eur Radiol. 2013;23:2061-2071.

14. Houssami N, Skaane P. Overview of the evidence on digital breast tomosynthesis in breast cancer detection. Breast (Edinburgh, Scotland). 2013; 22:101-108.

15. Skaane P, Bandos AI, Gullien R, et al. Comparison of digital mammography alone and digital mammography plus tomosynthesis in a population-based screening program. Radiology. 2013;267:47-56.

16. Friedewald SM, Rafferty EA, Rose SL, et al. Breast cancer screening using tomosynthesis in combination with digital mammography. JAMA. 2014;311:2499-2507.

17. Greenberg JS, Javitt MC, Katzen J, Michael S, Holland AE. Clinical performance metrics of 3D digital breast tomosynthesis compared with 2D digital mammography for breast cancer screening in community practice. AJR Am J Roentgenol. 2014;203:687-693.

18. Nelson HD, Tyne K, Naik A, Bougatsos C, Chan BK, Humphrey L. Screening for breast cancer: an update for the U.S. Preventive Services Task Force. Ann Intern Med. 2009;151(10):W237-W742.
19. Oeffinger KC, Fontham ETH, Etzioni R. Breast cancer screening for women at average risk. JAMA. 2015;314(15):1599-1614.

20. Centers for Disease Control. Breast cancer screening among adult women - behavioral risk factor surveillance system, United States, 2010. MMWR Morb Mortal Wkly Rep Suppl. 2012;61(02):46-50.

21. Centers for Disease Control and Prevention. Table 76. Use of Mammography Among Women Aged 40 and Over, by Selected Characteristics: United States, Selected Years 1987-2013. Health, United States, 2014. Washington, DC: CDC; 2014.

22. Caplan LS. To screen or not to screen: the issue of breast cancer screening in older women. Public Health Rev. 2001;29:231-240.

23. Deshpande AD, McQueen A, Coups EJ. Different effects of multiple health status indicators on breast and colorectal cancer screening in a nationally representative US sample. Cancer Epidemiol. 2012;36(3): 270-275.

24. Mor V, Pacala JT, Rakowski W. Mammography for older women: Who uses, who benefits? J Gerontol. 1992;47(SPEC. ISS.):43-49.

25. Yasmeen S, Hubbard RA, Romano PS, et al. Risk of advanced-stage breast cancer among older women with comorbidities. Cancer Epidemiol Biomarkers Prev. 2012;21(9):1510-1519.

26. Walter LC, Covinsky KE. Cancer screening in elderly patients: a framework for individualized decision making. JAMA. 2001;285: 2750-2756.

27. Walter LC, Schonberg MA. Screening mammography in older women: a review. JAMA. 2014;311:1336-1347.

28. Kiefe CI, Funkhouser E, Fouad MN, May DS. Chronic disease as a barrier to breast and cervical cancer screening. J Gen Intern Med. 1998; 13:357-365.

29. Wright PJ, Fortinsky RH, Covinsky KE, Anderson PA, Landefeld CS. Delivery of preventive services to older black patients using neighborhood health centers. J Am Geriatr Soc. 2000;48:124-130.

30. McBean AM, Yu X. The underuse of screening services among elderly women with diabetes. Diabetes Care. 2007;30:1466-1472.

31. Tan A, Kuo Y-F, Goodwin JS. Integrating age and comorbidity to assess screening mammography utilization. Am J Prev Med. 2012;42: 229-234.

32. Heflin MT, Oddone EZ, Pieper CF, Burchett BM, Cohen HJ. The effect of comorbid illness on receipt of cancer screening by older people. $J \mathrm{Am}$ Geriatr Soc. 2002;50:1651-1658.

33. Caban M, Kuo YF, Raji M, Tan A, Freeman J. Predictors of mammography use in older women with disability: the patients' perspectives. Med Oncol (Northwood, London, England). 2011;28(suppl 1): S8-S14.

34. Schonberg MA, McCarthy EP, Davis RB, Phillips RS, Hamel MB. Breast cancer screening in women aged 80 and older: results from a national survey. J Am Geriatr Soc. 2004;52:1688-1695.

35. Wu ZH, Black SA, Markides KS. Prevalence and associated factors of cancer screening: why are so many older Mexican American women never screened? Prev Med. 2001;33:268-273.

36. Reyes-Ortiz CA, Markides KS. Socioeconomic factors, immigration status, and cancer screening among Mexican American women aged 75 and older. Health Care Women Int. 2010;31:1068-1081.

37. Blustein J, Weiss LJ. The use of mammography by women aged 75 and older: factors related to health, functioning, and age. $J$ Am Geriatr Soc. 1998;46:941-946.

38. Ives DG, Lave JR, Traven ND, Schulz R, Kuller LH. Mammography and Pap smear use by older rural women. Public Health Rep. 1996; 111(3):244-250.

39. Scinto JD, Gill TM, Grady JN, Holmboe ES. Screening mammography: is it suitably targeted to older women who are most likely to benefit? J Am Geriatr Soc. 2001;49:1101-1104.

40. Schootman M, Jeffe DB. Identifying factors associated with disabilityrelated differences in breast cancer screening (United States). Cancer Causes Control. 2003;14:97-107.

41. Mayer JA, Slymen DJ, Drew JA, Wright BL, Elder JP, Williams SJ. Breast and cervical cancer screening in older women: the San Diego Medicare Preventive Health Project. Prev Med. 1992;21:395-404. 
42. Schonberg MA, Leveille SG, Marcantonio ER. Preventive health care among older women: missed opportunities and poor targeting. Am J Med. 2008;121:974-981.

43. Koya DL, Chen JG, Smith TG, Moran WP. Screening mammography use in Medicare beneficiaries reflects 4-year mortality risk. Am J Med. 2011;124:.e361-.e368

44. Walter LC, Lindquist K, Covinsky KE. Relationship between health status and use of screening mammography and Papanicolaou smears among women older than 70 years of age. Ann Intern Med. 2004;140: 681-688.

45. Schonberg MA, Breslau ES, McCarthy EP. Targeting of mammography screening according to life expectancy in women aged 75 and older. J Am Geriatr Soc. 2013;61:388-395.

46. Bynum JPW, Braunstein JB, Sharkey P, Haddad K, Wu AW. The influence of health status, age, and race on screening mammography in elderly women. Arch Intern Med. 2005;165:2083-2088.

47. Williams BA, Lindquist K, Sudore RL, Covinsky KE, Walter LC. Screening mammography in older women. Effect of wealth and prognosis. Arch Intern Med. 2008;168:514-520.

48. Mehta KM, Fung KZ, Kistler CE, Chang A, Walter LC. Impact of cognitive impairment on screening mammography use in older US women. Am J Public Health. 2010;100:1917-1923.

49. Thorpe JM, Kalinowski CT, Patterson ME, Sleath BL. Psychological distress as a barrier to preventive care in community-dwelling elderly in the United States. Med Care. 2006;44:187-191.

50. Sinclair N, Littenberg B, Geller B, Muss H. Accuracy of screening mammography in older women. AJR Am J Roentgenol. 2011;197: $1268-1273$.

51. Henderson LM, O’Meara ES, Braithwaite D, Onega T; Breast Cancer Surveillance Consortium. Performance of digital screening mammography among older women in the United States. Cancer. 2015;121(9): 1379-1386.

52. Mandelblatt JS, Tosteson AN, van Ravesteyn NT. Costs, evidence, and value in the medicare program: comment on "the cost of breast cancer screening in the medicare population". JAMA Intern Med. 2013;173:227-228.

53. Chiarelli AM, Edwards SA, Prummel MV, et al. Digital compared with screen-film mammography: performance measures in concurrent cohorts within an organized breast screening program. Radiology. 2013;268:684-693.

54. Smith-Bindman R, Kerlikowske K, Gebretsadik T, Newman J. Is screening mammography effective in elderly women? Am J Med. 2000;108: $112-119$.

55. Mandelblatt JS, Cronin KA, Bailey S, et al; Breast Cancer Working Group of the Cancer Intervention and Surveillance Modeling Network. Effects of mammography screening under different screening schedules: model estimates of potential benefits and harms. Ann Intern Med. 2009;151:738-747.

56. Randolph WM, Goodwin JS, Mahnken JD, Freeman JL. Regular mammography use is associated with elimination of age-related disparities in size and stage of breast cancer at diagnosis. Ann Intern Med. 2002;137:783-790.

57. Lee SJ, Boscardin WJ, Stijacic-Cenzer I, Conell-Price J, Sarah O, Walter LC. Time lag to benefit after screening for breast and colorectal cancer: Meta-analysis of survival data from the United States, Sweden, United Kingdom, and Denmark. BMJ (Online). 2013; 346:e8441.
58. Walter LC, Eng C, Covinsky KE. Screening mammography for frail older women: what are the burdens? J Gen Intern Med. 2001;16:779-784.

59. Alibhai SMH. Cancer screening: applying the evidence to adults beyond age 70. Geriatr Aging. 2006;9:164-171.

60. Walter LC, Lewis CL, Barton MB. Screening for colorectal, breast, and cervical cancer in the elderly: a review of the evidence. Am J Med. 2005;118:1078-1086.

61. Braithwaite D, Zhu W, Hubbard RA, et al; Breast Cancer Surveillance Consortium. Screening outcomes in older US women undergoing multiple mammograms in community practice: does interval, age, or comorbidity score affect tumor characteristics or false positive rates? J Natl Cancer Inst. 2013;105:334-341.

62. Fleming ST, Pursley HG, Newman B, Pavlov D, Chen K. Comorbidity as a predictor of stage of illness for patients with breast cancer. Med Care. 2005;43:132-140.

63. McPherson CP, Swenson KK, Lee MW. The effects of mammographic detection and comorbidity on the survival of older women with breast cancer. J Am Geriatr Soc. 2002;50:1061-1068.

64. Mandelblatt JS, Wheat ME, Monane M, Moshief RD, Hollenberg JP, Tang J. Breast cancer screening for elderly women with and without comorbid conditions. A decision analysis model. Ann Intern Med. 1992;116:722-730.

65. Lansdorp-Vogelaar I, Gulati R, Mariotto AB, et al. Personalizing age of cancer screening cessation based on comorbid conditions: model estimates of harms and benefits. Ann Intern Med. 2014;161:104-112.

66. Messecar DC. Mammography screening for older women with and without cognitive impairment. J Gerontol Nurs. 2000;26:14-24; quiz 52-13.

67. Charlson ME, Pompei P, Ales KL, MacKenzie CR. A new method of classifying prognostic comorbidity in longitudinal studies: development and validation. J Chronic Dis. 1987;40:373-383.

68. Mandelblatt J, Traxler M, Lakin P, Kanetsky P, Kao R. Mammography and Papanicolaou smear use by elderly poor black women. The Harlem Study Team. J Am Geriatr Soc. 1992;40:1001-1007.

69. Schousboe JT, Kerlikowske K, Loh A, Cummings SR. Personalizing mammography by breast density and other risk factors for breast cancer: analysis of health benefits and cost-effectiveness. Ann Intern Med. 2011; 155:10-20.

70. Walter LC. What is the right cancer screening rate for older adults. Arch Intern Med. 2011;171:2037-2039.

71. Schonberg MA, McCarthy EP, York M, Davis RB, Marcantonio ER. Factors influencing elderly women's mammography screening decisions: implications for counseling. BMC Geriatr. 2007;7:26.

72. Schonberg MA, Walter LC. Talking about stopping cancer screeningnot so easy: comment on "Older adults and forgoing cancer screening: 'I think it would be strange"'. JAMA Intern Med. 2013;173(7): 532-533.

73. Cruz M, Covinsky K, Widera EW, Stijacic-Cenzer I, Lee SJ. Predicting 10-year mortality for older adults. JAMA. 2013;309:874-876.

74. Schonberg MA, Hamel MB, Davis RB, et al. Development and evaluation of a decision aid on mammography screening for women 75 years and older. JAMA Intern Med. 2014;174:417-424.

75. Marcus PM, Freedman AN, Khoury MJ. Targeted cancer screening in average-risk individuals. Am J Prev Med. 2015;49(5):765-771.

76. Kramer BS, Elmore JG. Projecting the benefits and harms of mammography using statistical models: proof or proofiness? J Natl Cancer Inst. 2015;107(7).
Clinical Interventions in Aging

\section{Publish your work in this journal}

Clinical Interventions in Aging is an international, peer-reviewed journal focusing on evidence-based reports on the value or lack thereof of treatments intended to prevent or delay the onset of maladaptive correlates of aging in human beings. This journal is indexed on PubMed Central, MedLine,

\section{Dovepress}

CAS, Scopus and the Elsevier Bibliographic databases. The manuscript management system is completely online and includes a very quick and fair peer-review system, which is all easy to use. Visit http://www.dovepress. com/testimonials.php to read real quotes from published authors. 